\title{
Non Invasive Measurement of Hemoglobin using Optical Sensor
}

\author{
D. Sineka, S. Mythili
}

\begin{abstract}
Hemoglobin (hb) concentration is a basic parameter in the human blood, the absense of which causes anemia. The proposed work stays away from the superfluous wastage of blood while estimating hb concentration which could have serious impact on a anemic patient. There are fundamentally two classifications to be specific, invasive and non-invasive methods.In the invasive method blood sample is collected by hurtful needle stick. After that the collected blood sample is sent to a laboratory for examination. Then the results are announced back to the docter later which possibly leads to analysis and treatement delay. A non-invasive technique permits torment free online patient checking framework with least danger of infection. An optical non-invasive system is introduced for Hb focus. In this anew advanced system, retention of light by oxygenated and deoxygenated hemoglobin is estimated at two wavelengths $660 \mathrm{~nm}$ and 940nm. The specific wavelength of light is acquired from red and IR LED. The absorption coefficient of blood differs at various wavelength this reality is utilized to compute concentration of the hemoglobin.
\end{abstract}

Index Terms: hemoglobin concentration, LCD, Noninvasive, pic microcontroller, wavelength of light.

\section{INTRODUCTION}

Hemoglobin is nothing but the iron surrounding oxygen transport metallo protein in the red blood cells. The structure of hemoprotein sounds like quaternary structure, Associate in Nursing building of four compounds with right smart presence of Oxy hemoprotein and Deoxy hemoprotein. The red platelets count inadequacy in blood reduces its oxygen bringing capacity and denoted as Anemia. when anemia proceeds progressively, the indications are often in doubt and may include tired, a poor ability to work out. It must be critical before an individual ends up being discernibly pale. It is the most well-known blood disorder, affecting about thirty three percentage of the overall population. It impacts around one billion people. Anemia is more common in women than men, a youngsters and also in old age people. It rises the costs of medical treatment. Paleness goes undiscovered in various people and signs can be minor. When anemia goes severe the body may adapt for the lack of oxygen carrying capacity of the blood by raising cardiac output. In case it is left untreated it will causes numerous issues. So hb levels have to be estimated. By splitting red blood cell stable hemoglobin is attained in the diluent samples. Inthis, splitting of red blood cell is done with hemolysis process bringing dissolution of internal hemoglobin modern tools to determine hb in blood involves invasive method, in which the sample of blood is acquired from the subject by driving the finger of the same.

This invasive method uses needles causing direct contact with the blood which has the possibility for infection. A non cleaned outer condition incorporates numerous utility of a similar needle, encompassing temperature. Unpracticed professionals raise the likelihood of perpetrating contamination regarding the matters under evaluation. To address the constraints known within the existing systems, a non invasivehaemoglobin measurement system on Embedded technology was implemented in this paper.

\section{RELATED WORK}

A method which determine the haemoglobin concentration in blood donars which is based on non invasive pulse co oximetry. The goal of this examination is to approve the pulse co oximetry based haemoglobin prediction in blood donars[1]. In sedated sheep, the difference between invasive and a non invasive blood pressure measurements are taken. The objective of this examination was to decide the accuracy of the petMAP in anesthetized sheep confronting a medical procedure[2].Machine learning techniques and characteristic features of the photo plethysmography signals are utilized for the measurement of hemoglobin non-invasively[3].A noninvasive antenatal finding of hb Bart's disease by cardiothoracic ratio over the first period was given[4].The effects of a sympathetic block on Plethysmographic analysis of $\mathrm{Hb}$ concentration, Perfusion and Pleth variability Index with the Radical-7 apparatus was studied[5].The hb concentration in arterial blood was measured non-invasively. For this purpose a magnet was used which is set essentially over the radial artery of an individual whose hb concentration is to be discovered [6].Observable Red Laser light of wavelength $650 \mathrm{~nm}$ was used by the simple and beneficial noninvasive device for Blood Glucose Monitoring (RL-BGM)[7].The impacts of Skin thickness and Fingerprints was pretended to show the issues with Noninvasive RF Blood Glucose Sensing from Fingertips[8]. The Pulse Waves (PW) are portrayed non obtrusively from the upper and lower extremity from 52 healthy volunteers without known cardiovascular ailments[9].Dynamic Spectrum analysis is utilized for the estimation of hemoglobin concentration non obtrusively [10]. 


\section{METHODOLOGY}

The proposed system has a probe, a sensor setup, a pic micro controller connected to Liquid Crystal Display (LCD) and serial communication. Since the probe has to accommodate finger of the casuality of various finger cross section, a nominal diameter of $5 \mathrm{~cm}$ is preferred for the socket. Both the LEDs are placed inside the probe socket with photo diode in phase with the LEDs.IR LED and red LED are used as transmitter and photodiode is used as receiver. When supply of $5 \mathrm{v}$ when provided through voltage regulator to sensor circuit, probe is initiated. Depending on the HHB content in the blood, absorption of IR and red LED wavelength detection differs in photodiode. Photo diode works on the principle that the leakage current produced by the photo diode depends on the intensity of incident light.

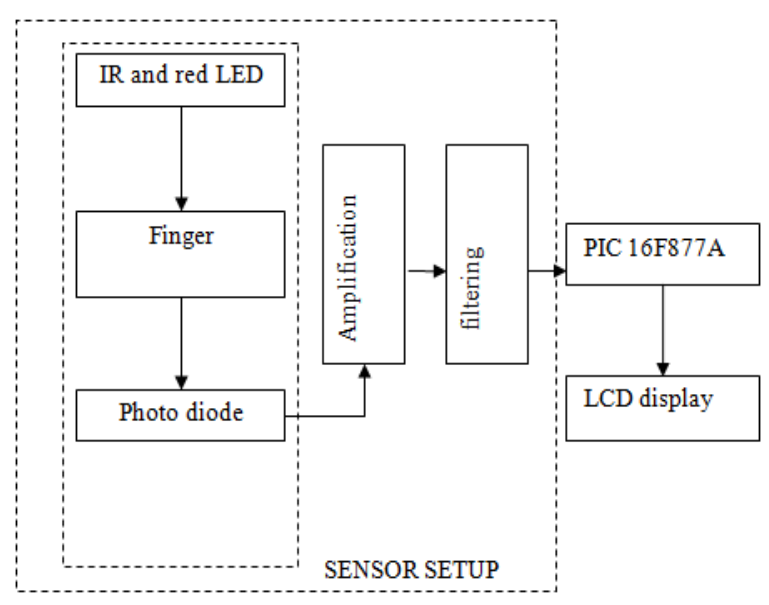

Fig 1.Block diagram

A considerable penetration and diffusion difference of light in red and near infrared region between oxygenated $\left[\mathrm{HbO}_{2}\right]$ and deoxygenated hemoglobin $[\mathrm{HHb}]$ is utilized for the principle estimation. Deoxygenated hemoglobin is optically much deeper to the red light $(600$ to $750 \mathrm{~nm}$ ) than Oxygenated hemoglobin. Where as the reverse is true in the near infrared region (900 to1000 nm). Therefore when HHB content is more RED LED wavelength is detected and vice versa. There variation in wavelength produce various current. The signal from the photodiode is low in amplitude so amplification of the signal is done to view the signal clearly. After that the signal is filtered for removing noise.PPG signal is then acquired by a microcontroller. By using MPLAB IDE, the code for measuring haemoglobin content is dumb into the pic Microcontroller (16F877A).Pic micro controller hence process the amplified signal obtained through sensor setup for obtaining hb count. LCD displays the desired result. Once the finger is inserted in the probe, microcontroller processes the sensor signal and provides the result in LCD display.

\section{RESULTS AND DISCUSSIONS}

Beer Lambert's law is used for developing the the absorbance notation in order to point out the absorption of light as a function of hbconcentration is denoted in the following equation.

$\mathrm{OD}=\log \left(\mathrm{I}_{0} / \mathrm{I}\right)=\varepsilon \mathrm{cl}$

Where,

$\mathrm{O}=$ Optial Density
$\mathrm{D}$

$\mathrm{I}_{0} \quad=\quad$ Incident light intensity

$\mathrm{I}=$ Transmitted light intensity

$\varepsilon=$ Extinction coefficient of hemoglobin

$\mathrm{c}=$ Concentration of haemoglobin

$1=$ Length of light path through the solution

Let,

$\mathrm{HbO}_{2}=\mathrm{A}$ and $\mathrm{HHb}=\mathrm{B}$ for simplicity.

Whenever estimated test has a mixure of oxygenated $\left[\mathrm{Hbo}_{2}\right]$ and deoxygenated $[\mathrm{HHb}]$ hemoglobin then equation (1) can be extended as,

$$
\mathrm{OD}^{\lambda}=\left\{\varepsilon_{B}^{\lambda}[\mathrm{B}]+\varepsilon_{A}^{\lambda}[\mathrm{A}]\right\} \mathrm{L}
$$

$\mathrm{OD}^{\lambda}$ - Optical density at wavelength $\lambda$

$\varepsilon_{B}^{\lambda} \quad$ - Extinction coefficient at wavelength $\lambda$

For molar concentration B

$\varepsilon_{A}^{\lambda} \quad$ - Extinction coefficient at wavelength $\lambda$

For molar concentration A

Assume path length $\mathrm{l}=1 \mathrm{~cm}$. Both oxygenated $\left[\mathrm{hbo}_{2}\right]$ and deoxygenated [hhb] haemoglobin concentrations can be measured at two specific wavelengths,

$$
\begin{aligned}
& {\left[H b o_{2}\right]=\frac{\varepsilon_{B}^{\lambda 2} * \mathrm{OD}^{\lambda 2}-\varepsilon_{\mathrm{B}}^{\lambda 1} \mathrm{OD}^{\lambda 2}}{\mathrm{~L}\left(\varepsilon_{\mathrm{B}}^{\lambda 2} * \varepsilon_{\mathrm{A}}^{\lambda 1}-\varepsilon_{\mathrm{B}}^{\lambda 1} * \varepsilon_{\mathrm{A}}^{\lambda 2}\right)}} \\
& {[\mathrm{HHb}]=\frac{\varepsilon_{\mathrm{A}}^{\lambda 2} * \mathrm{OD}^{\lambda 2}-\varepsilon_{\mathrm{A}}^{\lambda 1} \mathrm{OD} \mathrm{D}^{\lambda 2}}{L\left(\varepsilon_{\mathrm{B}}^{\lambda 1} * \varepsilon_{\mathrm{A}}^{\lambda 2}-\varepsilon_{\mathrm{B}}^{\lambda 2} * \varepsilon_{\mathrm{A}}^{\lambda 1}\right)}}
\end{aligned}
$$

Total $\mathrm{Hb}=[\mathrm{A}]+[\mathrm{B}]$

Hemoglobin concentration is calculated by utilizing the above equation and the observations are given in the following table,

Table I: Observation Results

\begin{tabular}{|l|l|l|l|}
\hline $\begin{array}{l}\text { S.no } \\
\text { (Male) }\end{array}$ & $\begin{array}{l}\text { Observed } \\
\text { Hemoglobin } \\
\text { concentration(gm/ } \\
\text { dl) }\end{array}$ & $\begin{array}{l}\text { S.no } \\
\text { (Female }\end{array}$ & $\begin{array}{l}\text { Observed } \\
\text { Hemoglobin } \\
\text { concentration(gm/ } \\
\text { dl) }\end{array}$ \\
\hline Subject1 & 11 & Subject1 & 10.7 \\
\hline Subject2 & 13 & Subject2 & 11.2 \\
\hline Subject3 & 11.9 & Subject3 & 9.8 \\
\hline Subject4 & 10.5 & Subject4 & 11 \\
\hline Subject5 & 12.5 & Subject5 & 11.8 \\
\hline Subject6 & 13 & Subject6 & 12 \\
\hline Subject7 & 11.9 & Subject7 & 12 \\
\hline Subject8 & 12.6 & Subject8 & 11 \\
\hline Subject9 & 11.8 & Subject9 & 10.9 \\
\hline Subject1 & 12.7 & $\begin{array}{l}\text { Subject1 } \\
0 \\
0\end{array}$ & 0 \\
\hline
\end{tabular}

The results indicates that the Hgb level can be calculated using this non invasive method.

\section{CONCLUSION}

In this, embedded system based non invasive device is proposed for estimation of haemoglobin concentration in blood by utilizing wavelength $660 \mathrm{~nm}$ and $940 \mathrm{~nm}$ which is obtained from RED and IR LED. Both the LED'S are utilized as a transmitter and the Photo diode is utilized as a 
receiver.The signal from the photo diode is low in amplitude so amplification process is done to view the signal clearly and noise in the signal can be removed by filtering process. After that the signal from the sensor setup is obtained by the microcontroller which process the amplified signal for the determination of haemoglobin concentration level. 20 subjects were taken for clinical experiment which includes 10 Male and 10 Female. The results indicate that the Hgb level can be derived by this approach noninvasively with acceptable precision and accuracy.The proposed non invasive technique permits torment free online patient observing with least danger of disease and diminished the patient inconvenience. The device does not require any operational expertise and it can be utilized by anyone all over the place.

\section{ACKNOWLEDGMENT}

The authors would like to thank the Management of PSNA College of Engineering and Technology, Dindigul for the support to complete this project inside the campus by providing Lab facility.

\section{REFERENCES}

1. Murtadha Al-Khabori , Arwa Z Al-Riyami, Khalil Al-Farsi , Mohamed Al-Huneini ,Hashim , Nasser Al-Kemyani , Shahina Daar," Validation of a non-invasive pulse CO-oximetry based hemoglobin estimation in normal blood donors" Transfusion and Apheresis Science ,vol.50, pp(95-98),2014.

2. Daniel Almeida, Michele Barletta , Lindsey Mathews, Lynelle Graham , Jane Quandt,"Comparison between invasive blood pressure and a non-invasive blood pressure monitor in anesthetized sheep" Research in Veterinary Science,vol.97,pp.(582-586),2014.

3. Kavsaoglu , Kemal Polat, M. Hariharan," Non-invasive prediction of hemoglobin level using machine learningtechniques with the PPG signal's characteristics features",Appliedsoft computing,vol.37,pp.983-991, 2015.

4. Li Zhen, Min Pan, Jin Han, Xin Yang, Yan-Mei Ou, Can Liao, Dong-Zhi Li," Non-invasive prenatal detection of haemoglobin Bart's disease by cardiothoracic ratio during the first trimester", European Journal of Obstetrics \& Gynecology and Reproductive Biology ,vol.193,pp(92-95), 2015.

5. C. Bergek, J. H. Zdolsek, and R. G. Hahn,” Non-invasive blood haemoglobin and plethysmographic variability index during brachial plexus block", British Journal of Anaesthesia,pp.(1-6),2015.

6. J.Rezuana Bai ,S.Mohanasankar,V.Jagadeesh Kumar,' Non-invasive Measurement of Hemoglobin Concentration Using Magnetic Plethysmo Gram",2016 IEEE international symposium on medical measurements and Applications(MeMeA),Benevento, Italy,2016.

7. H. Ali, F. Bensaali," Novel Approach to Non-Invasive Blood Glucose Monitoring based on Transmittance and Refraction of Visible Laser Light", access IEEE, vol.5, pp.9163-9174,may 2017.

8. Volkan Turgul, Izzet Kale," Simulating the Effects of Skin Thickness and Fingerprints to Highlight Problems with Non-Invasive RF Blood Glucose Sensing From Fingertips, IEEE sensors journal, vol. 17, no. 22, 2017.

9. Mikko Peltokangas, Antti Vehkaoja, Jarmo Verho, Ville M. Mattila, Pekka Romsi, Jukka Lekkala, and Niku Oksala," Age dependence of arterial pulse wave parameters extracted from dynamic blood pressure and blood volume pulse waves", IEEE Journal of Biomedical and Health Informatics, vol.21(1), pp.(142-149),2017.

10. Hiromitsu Furukaw,"Real-time multi-channel Fourier transform spectroscopy and Its application to non-invasive blood fat measurement, National Institute of AIST, Higashi 1-1-1, Tsukuba 305-8565, 2017.

\section{AUTHORS PROFILE}

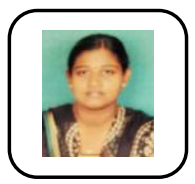

Sineka.D,Completed M.E. in Applied Electronics in PSNA College of Engineering and Technology, Dindigul,Tamilnadu,India. She Completed her B.E. degree in Electronics and Communication Engineering in SSM Institute of Engineering and Technology, Dindigul,Tamilnadu, India. Published a paper on Embedded Systems in an International journal. Field of interest includes Image processing and Embedded systems.

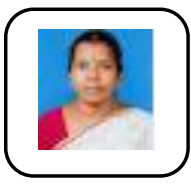

Dr.S.Mythiliis working as a Professor in the department of Electronics and Communication Engineering at PSNA College of Engineering and Technology,Dindigul,Tamilnadu,India. She Obtained her Ph.D.degree from Anna University(PT),ICE,in the year 2015.She completed her M.Tech degree in BME,IIT Madras in the year 2004. She completed her B.E. degree in Instrumentation and Control Engineering in 1995 from ArulmiguKalasalingam College of Engineering,Srivilliputhur,Tamilnadu,India.She has published more than 15 papers in both National and International journals.Her research interest includes Biomedical Instrumentation and BioSensors. 